\title{
Increasing Trend of Isolation of Non-Tuberculous Mycobacteria in a Tertiary University Hospital in South Korea
}

\author{
Jung-Wan Yoo, M.D. ${ }^{1}$, Kyung-Wook Jo, M.D. , Mi Na Kim, M.D. ${ }^{1}$, Sang Do Lee, M.D. ${ }^{1}$, Woo Sung Kim, M.D. \\ Dong Soon Kim, M.D. ${ }^{1}$, Tae Sun Shim, M.D. ${ }^{1}$ \\ ${ }^{1}$ Division of Pulmonary and Critical Care Medicine, ${ }^{2}$ Department of Laboratory Medicine, Asan Medical Center, University of \\ Ulsan College of Medicine, Seoul, Korea
}

\begin{abstract}
Background: The isolation of non-tuberculous mycobacteria (NTM) has been increasing in South Korea. To date, however, the cause of this increase has not been determined, and it remains unclear whether the use of liquid media has contributed to this increase. The aim of this study was to evaluate the factors associated with NTM isolation and the impact of liquid media on NTM culture.

Methods: Mycobacterial smear/culture results of respiratory specimens (sputum and bronchial aspirates), obtained during the years 2002, 2005, and 2010, were retrieved and analyzed retrospectively.

Results: During the years 2002, 2005, and 2010, 83,096 sputum specimens were collected from 31,104 patients, and were cultured for mycobacteria, using solid media only in the 2002 and 2005 specimens and both solid and liquid media in the 2010. Of these, 3,516 (4.2\%) specimens were smear-positive for acid-fast bacilli (AFB). The annual rate of NTM among positive culture specimens increased from 21\% in 2002 to $57.8 \%$ in 2010 (p $<0.001)$, as did the proportion of NTM, among AFB smear- and culture-positive specimens, from $12.2 \%$ in 2002 to $45.2 \%$ in $2010(\mathrm{p}<0.001)$. In 2010, the NTM culture rate was higher in the liquid than in the solid media $(13.9 \%$ vs. $8.4 \%, \mathrm{p}<0.001)$. The NTM rate among AFB-positive specimens was higher in patients aged $>50$ than $\leq 50$ years. Conclusion: The rate of NTM isolation has steadily been increasing at the hospital in South Korea, likely due in part to the use of liquid media for the culture.
\end{abstract}

Key Words: Nontuberculous Mycobacteria; Culture Media; Sputum; Republic of Korea

\section{Introduction}

Although the incidence and prevalence of non-tuberculous mycobacterial (NTM) isolation and disease have varied among populations, both have tended to increase over time ${ }^{1-6}$. In South Korea also, NTM isolation, using solid media, and disease have been increasing in clinical practice ${ }^{7,8}$. South Korea is a country with an intermediate tuberculosis (TB) burden, making it important

Address for correspondence: Tae Sun Shim, M.D. Division of Pulmonary and Critical Care Medicine, Asan Medical Center, University of Ulsan College of Medicine, 388-1, Pungnap-dong, Songpa-gu, Seoul 138-736, Korea Phone: 82-2-3010-3892, Fax: 82-2-3010-6968

E-mail: shimts@amc.seoul.kr

Received: Feb. 8, 2012

Revised: Feb. 21, 2012

Accepted: Apr. 17, 2012 to distinguish TB from NTM disease.

Liquid media is being used more commonly to isolate Mycobacterium tuberculosis and NTM?. Because the contamination rate is higher in liquid than in solid media, the combined use of liquid and solid media has been recommended for mycobacterial culture. We previously reported that the use of liquid media increased the isolation rate of mycobacteria, especially NTM, not just $M$. tuberculosis alone ${ }^{10}$. Thus, in addition to a real increase in NTM disease, the recent increase in NTM isolation rate may be due, at least in part, to the additional use of liquid media. At our institution, a tertiary referral hospital in South Korea, liquid media was introduced for mycobacterial culture in June 2007. To test the hypothesis that the increased rate of NTM isolation was partly due to the introduction of liquid media we determined the rate of $M$. tuberculosis/NTM isolation 
during 3 years, 2002, 2005, and 2010, and the effect of culturing in liquid media on this isolation rate.

\section{Materials and Methods}

\section{Data base}

Mycobacterial smear/culture results of respiratory specimens (sputum and bronchial aspirates) obtained during the years 2002, 2005, and 2010 were retrieved and analyzed retrospectively. The Asan Medical Center is a tertiary referral center in Seoul, Korea, with 2,200 beds in 2002 and 2005. In May 2008, 500 beds were added, giving a total of 2,700 beds in 2010. The patients with recovery of NTM in two or three consecutive year were included and counted separately each year.

\section{Specimen processing}

Sputum specimens were decontaminated with N-acetyl-L-cysteine in 5\% sodium hydroxide and concentrated by centrifugation at 3,000 $\times \mathrm{g}$ for 15 minutes. Acid-fast bacilli (AFB) smears were stained with fluorochrome, with positive results, defined as $>1 \sim 2$ AFB per 300 high-power field, confirmed by Ziehl-Neelsen staining.

Processed specimens were cultured only in 3\% Ogawa medium (Korean Institute of Tuberculosis, Seoul, Korea) in 2002 and 2005, and in both 3\% Ogawa medium and mycobacteria growth indicator tube medium (Becton Dickinson, Sparks, MD, USA) in 2010, with culture results monitored for 8 and 6 weeks, respectively. $M$. tuberculosis and NTM were distinguished by hybridization against a commercial molecular probe (AccuProbe; Gen-Probe Inc., San Diego, CA, USA) or duplex PCR (Molecules and Diagnostics Inc., Wonju, Korea). NTM was identified using a polymerase chain reaction-restriction fragment length polymorphism method, based on the $r p o B$ gene, at the Korean Institute of Tuberculosis and Asan Medical Center ${ }^{11}$. The study protocol was approved by the Institutional Review Board of the Asan Medical Center.

\section{Statistical analysis}

Data are expressed as numbers (percentage) or mean and standard deviation. Categorical variables were analyzed using Pearson's Chi-square test. Numerical variables were analyzed using one-way ANOVA, followed by a Bonferroni post-hoc test. Cochrane Armitage Trend test was performed using for trend analysis. A p-value $<0.05$ was considered statistically significant. All statistical analyses were performed using SPSS version 12.0 (SPSS Inc., Chicago, IL, USA) and SAS version 9.1 (SAS Institute, Cary, NC, USA).

\section{Results}

\section{Patient characteristics and culture of specimens in solid media}

During the 3 years of this study, 83,096 sputum specimens were collected from 31,104 patients and cultured for mycobacteria, with 3,516 (4.2\%) specimens being smear-positive for AFB (Table 1). M. tuberculosis or NTM was observed in 7,122 (8.6\%) sputum specimens cultured in solid media. The mean patient age was $56.7 \pm 15.6$ years and $60.1 \%$ were male. The mean age in 2010 was significantly higher than other years ( $p$ $<0.001$ ) and was not different between 2002 and 2010 $(\mathrm{p}=0.992)$. The male proportion in 2010 was significantly lower compared to that of 2002 or 2005 (p $<0.01)$ and was not different between 2002 and 2005 $(\mathrm{p}=0.320)$. The recovery rate of NTM from specimens and proportion of patients with NTM isolation increased from $21 \%$ in 2002 to $57.8 \%$ in 2010 and from $30.2 \%$ in 2002 to $62.9 \%$ in 2010, respectively (each $\mathrm{p}<0.001$ ) (Table 1, Figure 1). The proportion of AFB smear-positive and culture-positive specimens positive for NTM also increased, from $12.2 \%$ in 2002 to $45.2 \%$ in 2010 (p $<0.001$ ) (Table 1). In contrast, the rate of $M$. tuberculosis isolation declined over time.

\section{Solid vs. liquid media}

Both solid and liquid media were used to isolate mycobacteria from 31,615 sputum specimens during 2010 (Tables 2, 3). Of these, 4,723 (14.9\%) specimens were positive for $M$. tuberculosis or NTM. The positive-culture rate was higher in liquid than in solid media (13.9\% 
Table 1. Baseline characteristics of culture results by solid media

\begin{tabular}{|c|c|c|c|c|}
\hline & $2002(\%)$ & $2005(\%)$ & $2010(\%)$ & $p$-value* \\
\hline No. of specimens & 26,820 & 24,661 & 31,615 & \\
\hline No. of patients & 9,821 & 9,655 & 11,628 & \\
\hline Age, yr $( \pm S D)$ & $55.6 \pm 15.7$ & $55.6 \pm 15.8$ & $58.5 \pm 15.1$ & $<0.001^{\dagger}$ \\
\hline Gender, male & 6,026 (61.4) & $5,857(60.7)$ & $6,280(58.7)$ & $<0.01^{\ddagger}$ \\
\hline $\mathrm{AFB}(+)$ & $1,283(4.8)$ & $922(3.7)$ & $1,311(4.1)$ & $<0.001$ \\
\hline Positive culture specimens & $1,921(7.2)$ & $2,553(10.4)$ & $2,648(8.4)$ & 0.003 \\
\hline Mycobacterium tuberculosis & $1,518(79.0)$ & $1,230(48.2)$ & $1,118(42.2)$ & $<0.001$ \\
\hline NTM & $403(21.0)$ & $1,323(51.8)$ & $1,530(57.8)$ & \\
\hline Positive culture patients & $873(8.9)$ & $1,344(13.9)$ & $1,104(9.5)$ & 0.38 \\
\hline M. tuberculosis & $609(69.8)$ & $506(37.6)$ & $410(37.1)$ & $<0.001$ \\
\hline NTM & $264(30.2)$ & $838(62.4)$ & $694(62.9)$ & \\
\hline AFB smear $(+) /$ Culture $(+)$ specimens & $801(3.0)$ & $677(2.7)$ & $933(3.0)$ & 0.951 \\
\hline M. tuberculosis & $703(87.8)$ & $518(76.5)$ & $511(54.8)$ & $<0.001$ \\
\hline NTM & $98(12.2)$ & $159(23.5)$ & $422(45.2)$ & \\
\hline
\end{tabular}

${ }^{*}$ One-Way ANOVA was done to compare numberical values between three groups. ${ }^{\dagger}$ Compared with mean age in $2010 .{ }^{\dagger}$ Compared with gender proportion in 2010.

AFB: acid-fast bacilli; NTM: non-tuberculous mycobacteria.

A

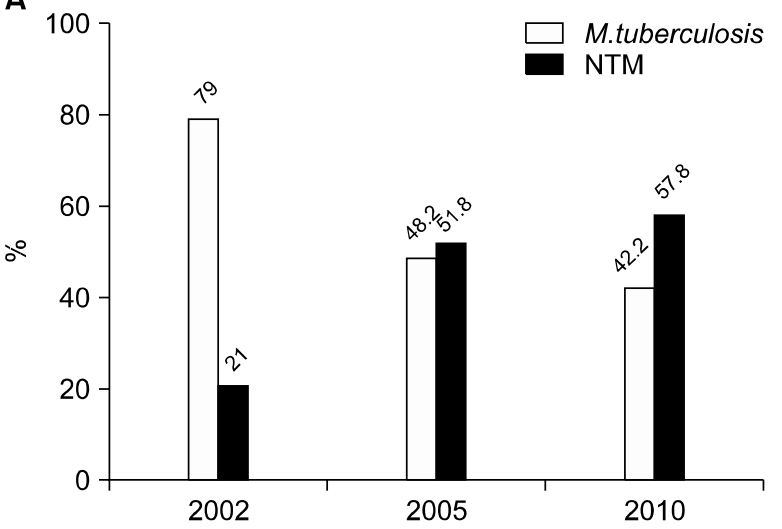

B

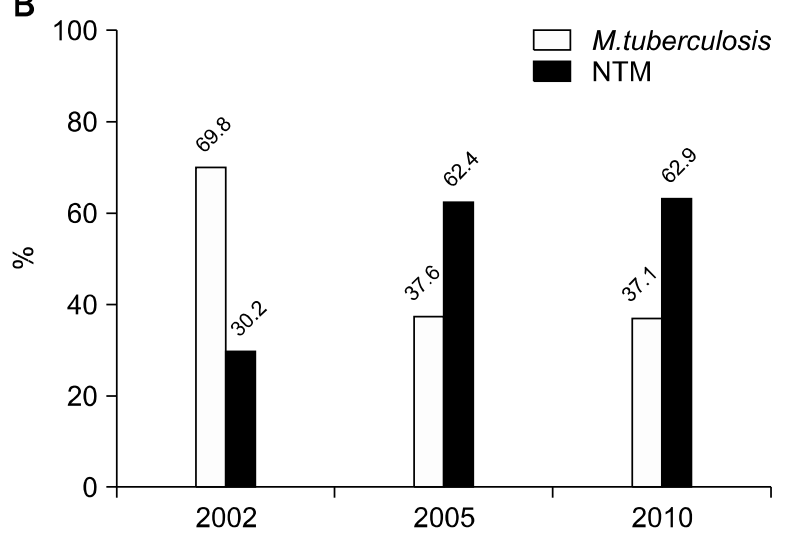

Figure 1. Recovery rate of Mycobacterium tuberculosis and non-tuberculous mycobacteria (NTM) on solid media in 2002, 2005, and 2010 ( $p<0.001)$. (A) Specimens. (B) Patients.

vs. $8.4 \%, \mathrm{p}<0.001)$; for example, $30.4 \%(489 / 1,607)$ of the $M$. tuberculosis and $50.9 \%(1,586 / 3,116)$ of the NTM isolates were cultured only in liquid media. However, among 1,179 (3.7\%) AFB smear-positive/culture-positive specimens, the proportions of $M$. tuberculosis and NTM did not differ between solid and liquid media.

\section{Factors predictive of NTM in AFB smear-positive specimens}

Table 4 shows the distributions of $M$. tuberculosis and NTM in AFB smear-positive/culture-positive specimens $^{12}$. During all three years, the proportion of NTM by gender was similar but $M$. tuberculosis isolation was associated with higher AFB smear grade. However, at all AFB smear grades, the proportion with NTM increased over time. The rate trend of NTM isolation was higher as patients were older ( $p$ for trend $<0.001$ ), especially the rate of NTM positivity was higher in patients aged $>50$ years, whereas NTM was rare and the rate stable in patients aged <30 years. During 2010, however, the rate of NTM did not differ between solid and 
JW Yoo et al: Increasing trend of NTM isolation

Table 2. Comparison of solid and liquid media in 2010

\begin{tabular}{|c|c|c|c|}
\hline & Solid media (\%) & Liquid media (\%) & Combined $^{*}(\%)$ \\
\hline No. of specimens & $31,615(100)$ & $31,615(100)$ & $31,615(100)$ \\
\hline No. of patients & $11,628(100)$ & $11,628(100)$ & $11,628(100)$ \\
\hline Positive culture specimens & $2,648(8.4)$ & 4,389 (13.9) & $4,723(14.9)$ \\
\hline Mycobacterium tuberculosis & $1,118(42.2)$ & $1,554(35.4)$ & 1,607 (34) \\
\hline NTM & $1,530(57.8)$ & $2,835(64.6)$ & $3,116(66)$ \\
\hline Positive culture patients & $1,104(9.5)$ & $1,893(16.3)$ & $2,052(17.6)$ \\
\hline M. tuberculosis & $410(37.1)$ & $532(28.1)$ & $545(26.6)$ \\
\hline NTM & $694(62.9)$ & $1,361(71.9)$ & $1,507(73.4)$ \\
\hline Smear $(+) /$ Culture $(+)$ specimens & $933(3.0)$ & $1,160(3.7)$ & $1,179(3.7)$ \\
\hline M. tuberculosis & $511(54.8)$ & $634(54.7)$ & $637(54.0)$ \\
\hline NTM & $422(45.2)$ & $526(45.3)$ & $542(46.0)$ \\
\hline Smear $(+) /$ Culture $(+)$ patients & $351(3.0)$ & $410(3.5)$ & $418(3.6)$ \\
\hline M. tuberculosis & $193(54.9)$ & $221(53.9)$ & $223(53.3)$ \\
\hline NTM & $158(45.1)$ & $189(46.1)$ & $195(46.7)$ \\
\hline
\end{tabular}

*Either test positive by solid media or by liquid media is regarded as positive results.

NTM: non-tuberculous mycobacteria.

Table 3. Recovery rate of solid and liquid media in 2010

\begin{tabular}{|c|c|c|c|}
\hline \multirow{2}{*}{ Culture system } & \multicolumn{3}{|c|}{ No. (\%) positive cultures for } \\
\hline & Mycobacterium tuberculosis $(n=1,607)$ & NTM $(n=3,116)$ & All mycobacteria $(n=4,723)$ \\
\hline Solid media only & $53(3.3)$ & $281(9.1)$ & $334(7.0)$ \\
\hline Liquid media only & $489(30.4)$ & $1,586(50.9)$ & $2,075(44.0)$ \\
\hline Both media & 1,065 (66.3) & $1,249(40.0)$ & 2,314 (49.0) \\
\hline
\end{tabular}

NTM: non-tuberculous mycobacteria.

liquid media according to age and AFB-smear status (data not shown).

\section{Discussion}

Although previous studies showed that NTM disease is increasing worldwide and that the incidence of NTM isolation is higher in liquid than in solid media, no reports to date have shown both findings. Our findings suggested that the recent increase in NTM isolation in South Korea, a country with an intermediate TB burden, may be due to both the use of liquid media and a real increase in the incidence of NTM. Moreover, the increased trend of NTM isolation was associated with patients >50 years. The analysis after excluding overlapped patients with NTM isolation showed the similar results in the studied periods. The trend of increasing NTM isolation was also observed when subgroup analysis by gender separately was performed (data not shown). Therefore, a diagnostic algorithm involving nucleic acid amplification (NAA) tests may be used to rapidly distinguish $M$. tuberculosis from NTM in AFB smear-positive specimens, especially those of subjects aged $>50$ years, even in a country in which $\mathrm{TB}$ is prevalent.

NTM isolation and disease have tended to increase worldwide over time. For example, a study from Ontario showed increased recovery over time in patients with pulmonary NTM, from 9.1 per 100,000 population in 1997 to 14.1 per 100,000 in 2003, while, at the same time, the incidence of TB declined ${ }^{1}$. Studies from Denmark and Netherlands have also reported increasing 
Table 4. Distributions of Mycobacterium tuberculosis and NTM according to gender, AFB smear status and age groups in AFB smear-positive/culture-positive specimens using solid media

\begin{tabular}{|c|c|c|c|c|c|c|}
\hline & \multicolumn{6}{|c|}{ Year } \\
\hline & \multicolumn{2}{|l|}{2002} & \multicolumn{2}{|l|}{2005} & \multicolumn{2}{|l|}{2010} \\
\hline & M. tuberculosis (\%) & NTM (\%) & M. tuberculosis (\%) & NTM (\%) & M. tuberculosis (\%) & NTM (\%) \\
\hline No. & 304 & 51 & 173 & 50 & 193 & 158 \\
\hline Male, n (\%) & $195(64.1)$ & $24(47.1)$ & 111 (64.2) & $26(52.0)$ & $118(61.1)$ & $80(50.6)$ \\
\hline \multicolumn{7}{|c|}{ AFB $^{\star}$ smear $(+)$} \\
\hline Trace & $60(73.2)$ & $22(26.8)$ & $75(66.4)$ & $38(33.6)$ & $97(46.9)$ & $110(53.1)$ \\
\hline $1+$ & 221 (86) & $36(14)$ & $118(67)$ & $58(33.0)$ & $197(50.6)$ & $192(49.4)$ \\
\hline $2+$ & $151(84.4)$ & $28(15.6)$ & $111(77.1)$ & $33(22.9)$ & $105(61.8)$ & $65(38.2)$ \\
\hline $3+$ & $74(93.7)$ & $5(6.3)$ & $117(84.8)$ & $21(15.2)$ & $78(65.5)$ & $41(34.5)$ \\
\hline $4+$ & 197 (96.6) & $7(3.4)$ & $97(91.5)$ & $9(8.5)$ & $34(70.8)$ & $14(29.2)$ \\
\hline \multicolumn{7}{|l|}{$\mathrm{Age}^{\dagger}$} \\
\hline $0 \sim 10$ & 0 & 0 & 0 & 0 & 0 & 0 \\
\hline $11 \sim 20$ & $13(100)$ & 0 & $21(100)$ & 0 & $7(100)$ & 0 \\
\hline $21 \sim 30$ & $85(90.4)$ & $9(9.6)$ & $73(98.6)$ & $1(1.4)$ & $55(98.2)$ & $1(1.8)$ \\
\hline $31 \sim 40$ & $86(94.5)$ & $5(5.5)$ & $98(91.6)$ & $9(8.4)$ & $76(83.5)$ & $15(16.5)$ \\
\hline $41 \sim 50$ & $134(93.7)$ & $9(6.3)$ & 102 (72.3) & $39(27.7)$ & $107(72.8)$ & $40(27.2)$ \\
\hline $51 \sim 60$ & $124(87.9)$ & $17(12.1)$ & $59(61.5)$ & $37(38.5)$ & $115(42.4)$ & $156(57.6)$ \\
\hline $61 \sim 70$ & $139(82.7)$ & $29(17.3)$ & $97(65.5)$ & $51(34.5)$ & 65 (38) & $106(62)$ \\
\hline$>70$ & $122(80.8)$ & $29(19.2)$ & $68(75.6)$ & $22(24.4)$ & $86(45.0)$ & 105 (55) \\
\hline
\end{tabular}

${ }^{*}$ AFB smear results were reported quantitatively according to American Thoracic Society guidelines ${ }^{12} .{ }^{\dagger} p$ for trend $<0.001$. NTM: non-tuberculous mycobacteria; AFB: acid-fast bacilli.

rates of NTM disease ${ }^{2,6}$, and studies from several Asian countries have shown increases in the incidence of NTM lung disease $e^{3,5}$. In Taiwan, where the incidence of TB remains high, the proportion of patients with NTM increased from $32.3 \%$ in 2000 to $49.8 \%$ in $2008^{4}$. Also, in Korea the rates of NTM isolation and NTM-associated lung disease have increased. For example, the rate of NTM isolation from sputum specimens obtained at a tertiary-care medical center in South Korea was 31.7\%, and the NTM recovery rate from AFB smear-positive sputum specimens was high ${ }^{7}$. From 2002 to 2008, the number of patients with NTM lung diseases increased, while the number of TB patients declined ${ }^{8}$.

The reasons for increased recovery of NTM are not yet clear. In Korea, the increases in NTM isolation and disease seem to be marked. We found that, among AFB smear-positive/culture-positive specimens, the percentage containing NTM increased markedly, from $12.2 \%$ in 2002 to $45.2 \%$ in 2010 . This increase may be due to improvements in laboratory culture methods, increased awareness of NTM, a larger proportion of patients aged $>50$ years, an increased prevalence of predisposing factors such as chronic obstructive lung disease (COPD) and, possibly, increased environmental exposure ${ }^{13,14}$. The prevalence of COPD, an important risk factor for NTM, is high ${ }^{15}$, and the population of Korea is aging. These factors, however, do not seem to account entirely for the increases in NTM isolation and disease rates. Since we only investigated mycobacteriologic results, we could not know whether isolated NTM were pathogen or colonizer in our study.

Both solid and liquid media have been recommended for mycobacterial culture ${ }^{10,16}$. In agreement with previous findings ${ }^{10}$, we found that culture in liquid media increased the rate of mycobacterial isolation, especially of NTM. Although liquid media is associated with a high rate of contamination, we found no difference in the contamination rate between solid and liquid media. 
Since liquid media are increasingly used for mycobacterial culture in Korea, the increased rate of NTM recovery may be due, at least in part, to increased culture in liquid media. Hence, in comparing NTM isolation rates, it is important to compare results using the same culture media.

The NTM recovery rates were higher in elderly than in young patients with $\mathrm{AFB}$ smear-positive results. Positivity on AFB smear has been considered a surrogate marker for a diagnosis of $\mathrm{TB}$ and contact with AFB-positive subjects has been considered a risk factor for TB. If AFB-positive results are caused by NTM rather than by $M$. tuberculosis, investigation of contacts is unnecessary. Rapid identification techniques, such as NAA tests, should be used to differentiate $M$. tuberculosis from NTM in AFB smear-positive sputum samples, even in an intermediate TB-burden country such as South Korea ${ }^{12,17}$.

This study had several limitations, including its performance at a single tertiary referral center. Therefore, selection bias cannot be excluded and our study subjects may not represent the general population. In addition, we collected and analyzed only laboratory results, and did not evaluate clinical characteristics, including the presence of true NTM disease. Furthermore, we did not determine the NTM species isolated.

In conclusion, we found that the rate of NTM isolation from sputum specimens has steadily increased in a hospital in South Korea, an increase due in part to the increased use of liquid media. As in the US, NAA tests should be performed to rapidly distinguish $M$. tuberculosis from NTM in AFB smear-positive sputum samples, especially for elderly subjects, in South Korea.

\section{References}

1. Marras TK, Chedore P, Ying AM, Jamieson F. Isolation prevalence of pulmonary non-tuberculous mycobacteria in Ontario, 1997-2003. Thorax 2007;62:661-6.

2. van Ingen J, Bendien SA, de Lange WC, Hoefsloot W, Dekhuijzen PN, Boeree MJ, et al. Clinical relevance of non-tuberculous mycobacteria isolated in the Nijme-
gen-Arnhem region, The Netherlands. Thorax 2009;64: 502-6.

3. Hosker HS, Lam CW, Ng TK, Ma HK, Chan SL. The prevalence and clinical significance of pulmonary infection due to non-tuberculous mycobacteria in Hong Kong. Respir Med 1995;89:3-8.

4. Lai CC, Tan CK, Chou CH, Hsu HL, Liao CH, Huang $\mathrm{YT}$, et al. Increasing incidence of nontuberculous mycobacteria, Taiwan, 2000-2008. Emerg Infect Dis 2010; 16:294-6.

5. Tsukamura M, Kita N, Shimoide H, Arakawa H, Kuze A. Studies on the epidemiology of nontuberculous mycobacteriosis in Japan. Am Rev Respir Dis 1988;137: 1280-4.

6. Andréjak C, Thomsen VØ, Johansen IS, Riis A, Benfield TL, Duhaut P, et al. Nontuberculous pulmonary mycobacteriosis in Denmark: incidence and prognostic factors. Am J Respir Crit Care Med 2010;181: 514-21.

7. Jeon K, Koh WJ, Kwon OJ, Suh GY, Chung MP, Kim $\mathrm{H}$, et al. Recovery rate of NTM from AFB smear-positive sputum specimens at a medical centre in South Korea. Int J Tuberc Lung Dis 2005;9:1046-51.

8. Park YS, Lee CH, Lee SM, Yang SC, Yoo CG, Kim YW, et al. Rapid increase of non-tuberculous mycobacterial lung diseases at a tertiary referral hospital in South Korea. Int J Tuberc Lung Dis 2010;14:1069-71.

9. Chien HP, Yu MC, Wu MH, Lin TP, Luh KT. Comparison of the BACTEC MGIT 960 with Löwenstein-Jensen medium for recovery of mycobacteria from clinical specimens. Int J Tuberc Lung Dis 2000;4:866-70.

10. Bae E, Im JH, Kim SW, Yoon NS, Sung H, Kim MN, et al. Evaluation of combination of BACTEC mycobacteria growth indicator tube 960 system and Ogawa media for mycobacterial culture. Korean J Lab Med 2008; 28:299-306.

11. Devallois A, Goh KS, Rastogi N. Rapid identification of mycobacteria to species level by PCR-restriction fragment length polymorphism analysis of the hsp65 gene and proposition of an algorithm to differentiate $34 \mathrm{my}-$ cobacterial species. J Clin Microbiol 1997;35:2969-73.

12. Diagnostic Standards and Classification of Tuberculosis in Adults and Children. This official statement of the American Thoracic Society and the Centers for Disease Control and Prevention was adopted by the ATS Board of Directors, July 1999. This statement was endorsed by the Council of the Infectious Disease Society of America, September 1999. Am J Respir Crit Care Med 2000;161(4 Pt 1):1376-95. 
13. Glassroth J. Pulmonary disease due to nontuberculous mycobacteria. Chest 2008;133:243-51.

14. Daley CL, Griffith DE. Pulmonary non-tuberculous mycobacterial infections. Int J Tuberc Lung Dis 2010;14: 665-71.

15. Yoo KH, Kim YS, Sheen SS, Park JH, Hwang YI, Kim $\mathrm{SH}$, et al. Prevalence of chronic obstructive pulmonary disease in Korea: the fourth Korean National Health and Nutrition Examination Survey, 2008. Respirology
2011;16:659-65.

16. Bird BR, Denniston MM, Huebner RE, Good RC. Changing practices in mycobacteriology: a follow-up survey of state and territorial public health laboratories. J Clin Microbiol 1996;34:554-9.

17. Centers for Disease Control and Prevention (CDC). Updated guidelines for the use of nucleic acid amplification tests in the diagnosis of tuberculosis. MMWR Morb Mortal Wkly Rep 2009;58:7-10. 